\title{
Production and Development status of MPPC
}

\author{
Kazuhisa Yamamura ${ }^{1}$ \\ Solid State Division, Hamamatsu Photonics K.K. \\ Hamamatsu-City, 435-8558 Japan iliation \\ E-mail: yamamura@ssd.hpk.co.jp \\ Kenichi Sato, Shogo Kamakura \\ Solid State Division, Hamamatsu Photonics K.K. \\ Hamamatsu-City, 435-8558 Japan iliation
}

\section{Shinji Ohsuka}

Central Research Laboratory, Hamamatsu Photonics K.K.

Hamamatsu-City, 434-8601 Japan ffiliation

The MPPC (Multi-Pixel Photon Counter) is a solid state device made up of multiple APD (Avalanche Photo Diode) pixels operated in Geiger mode. MPPC have excellent photon counting capability at room temperature. Hamamatsu Photonics released single channel devices having the active area of $1 \times 1 \mathrm{~mm}$ and $3 \times 3 \mathrm{~mm}$ and array devises having 4 or 16 channels. And we are developing low noise MPPC with a thermoelectrically cooler. As a good blue sensitivity, MPPC is well matched to detect the week emission light of scintillators for HEP or medical applications. For example, T2K experiment adopted MPPCs, and we have delivered $60 \mathrm{Kpcs}$ of $1.3 \times 1.3 \mathrm{~mm}-\mathrm{MPPCs}$. In mass production, we confirmed that the characteristic of MPPCs are very uniform between lot to lot. And as a good timing resolution and insensitive to magnetic field, MPPC is expected to use for the MRI-PET and TOF-PET detectors. For PET application, we are now evaluating energy resolution and timing resolution of $3 \times 3 \mathrm{~mm}$ MPPC coupled with LYSO. And we are measuring the response characteristic of each pixels in MPPC chip and studying the relationship between resistance of trace electrode and timing resolution. The evaluation result will be explained.

International Workshop on New Photon Detectors (PD09)

Shinshu University Matsumoto Japan

24-26 June 2009

$1 \quad$ Speaker 


\section{Introduction}

The novel solid-state photon counting device called SiPM in general has been developed in Russia. The MPPC is a kind of SiPM family, but the structure is based on Hamamatsu Avalanche Photo Diode (APD) adopted by high energy experiment calorimeter. The features of MPPC are high gain $\left(10^{5}\right.$ to $\left.10^{6}\right)$, low bias voltage operation $(<100 \mathrm{~V})$, room temperature operation, low dark count rate $(<1 \mathrm{MHz} / \mathrm{mm} 2)$, high Photon Detection Efficiency (PDE), superior time resolution $(<<1 \mathrm{~ns})$, insensitive to magnetic field, low power consumption and mechanical robustness. These features will be suitable for MRI-PET, TOF-PET, High energy physics experiment, astronomy, fluorescence measurement, DNA BIO-chip sequencer and environmental analysis [1] [2] [3] [4]. One of the most notable feature of MPPC is the good blue sensitivity by adapting the structure (we call "HPK Reverse Structure"), and match to detect emission light of popular scintillators [5].

T2K experiment adopted MPPCs, and we have delivered $60 \mathrm{Kpcs}$ of $1.3 \mathrm{~mm} \square$-MPPCs without shipment problem. Timing resolution is the important future for PET application, we are now studying new MPPC for improving timing resolution.

\section{Line up of MPPCs [6]}

Figure 1 shows the line up of Hamamatsu MPPC. S10362-11 series is the size of $1 \mathrm{~mm}$ active area, and are three type of pixel pitch, 25um (1600 pixels), 50um (400 pixels), 100um (100 pixels). In addition, there are three type of package, CAN-type(U type), ceramic type (C type), SMD type (P type). Figure 2 shows the wavelength dependence of PDE(Photo Detection Efficiency, including the cross-talk and after pulse) of each type. The peak wavelength is around 440nm, and match to the general scintillator emission wavelength. Due to the nature of the package, at the less than $350 \mathrm{~nm}$, SMD type (P type) is smaller sensitivity than other package products. The recommended gain of 25,50,100 um pixel pitch MPPCs are $2.75 \times 10^{5}, 7.5 \times 10^{5}, 2.4 \times 10^{6}$ respectively. As gain is so sensitive to voltage and temperature, the operating voltage (Vop) and the temperature must be controlled. Figure 3 and 4 shows the gain dependent of Vop and temperature respectively. For example 50um pixel size which is, approximately $73 \% / 1 \mathrm{~V}$, approximately $4 \% /{ }^{\circ} \mathrm{C}$ of the variation.

For $3 \mathrm{~mm} \square$ active area, there are $\mathrm{S} 10362-33$ series (ceramic type) and S10931 series (SMD type), and a pixel pitch of 25um (14400 pixels), 50um (3600 pixels), 100um (900 pixels). Assuming the use of PET, Combining S10362-050C with LYSO scintillator, we have obtained energy resolution of $11.8 \%$ (FWHM) and time resolution of 410ps (FWHM) for $511 \mathrm{KeV} \gamma$-ray. (Figures 5 and 6)

For MPPC array, there are S10984 series (1mm $\square-1 \mathrm{x} 4 \mathrm{ch})$ and $\mathrm{S} 10985$ series (3mm $\square$ $2 \times 2 \mathrm{ch})$. These are monolithic arrays without gap between channels. S11064 series is the $4 \times 4$ ch discrete array mounted 16 pieces of S10931 equivalent MPPCs on PCB. It is the merit that we can choose 16 devises whose characteristic are uniform. Figure 7 shows gain 
uniformity of $3.5 \sim 7 \%(25 \mathrm{um})$ and $2.5 \sim 13.5 \%(50 \mathrm{um})$ respectively. As each 16 channels have anode and cathode terminals, it can also be adjusted the voltage of channels separately.

S11028 series is the $1 \mathrm{~mm} \square$-MPPC with a thermoelectrically cooler and temperature sensor in the TO-8 package. In use at room temperature and control MPPC temperature at $-10^{\circ} \mathrm{C}$, it can be used about $1 / 20$ in dark count.

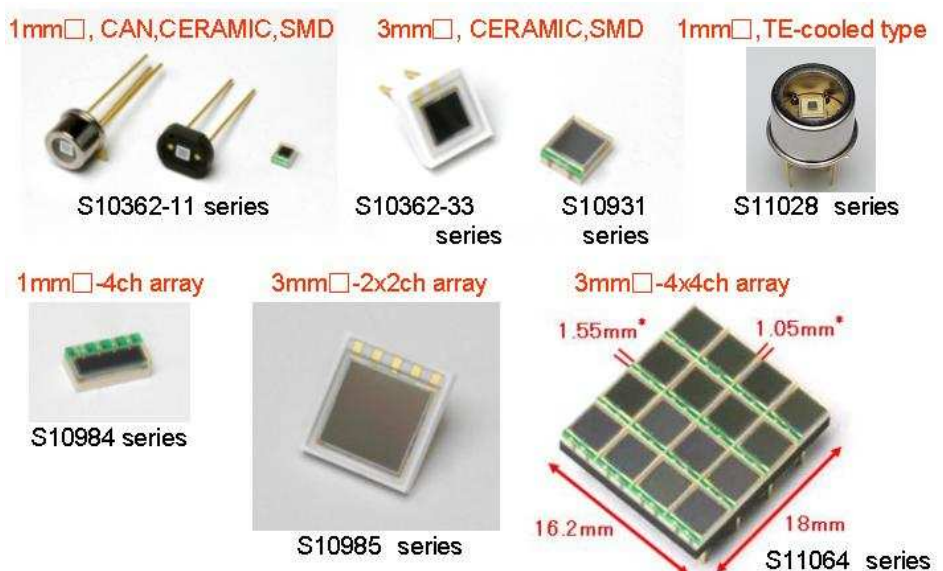

Fig.1 Line up of MPPCs

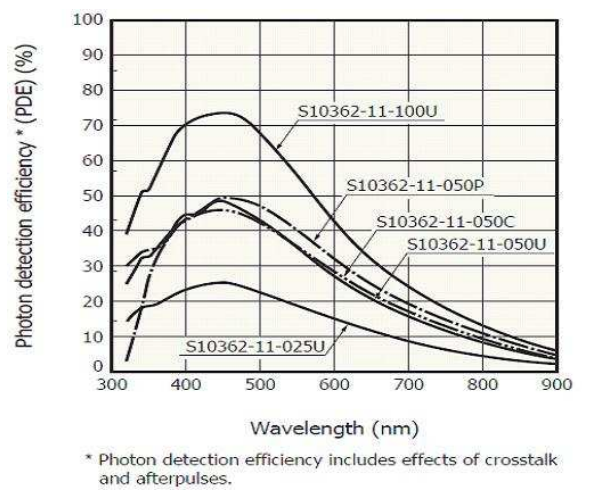

Fig.2 PDE vs. wavelength

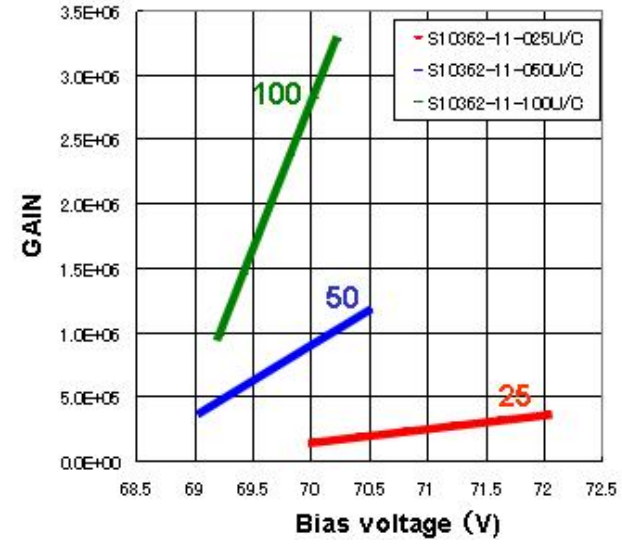

Fig.3 Gain vs. bias voltage

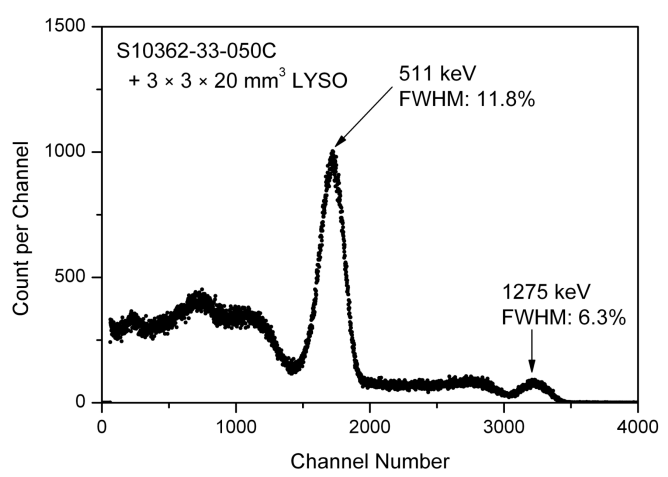

Fig.5 Energy resolution of $3 \mathrm{~mm} \square$-MPPC coupled with LYSO scintillator

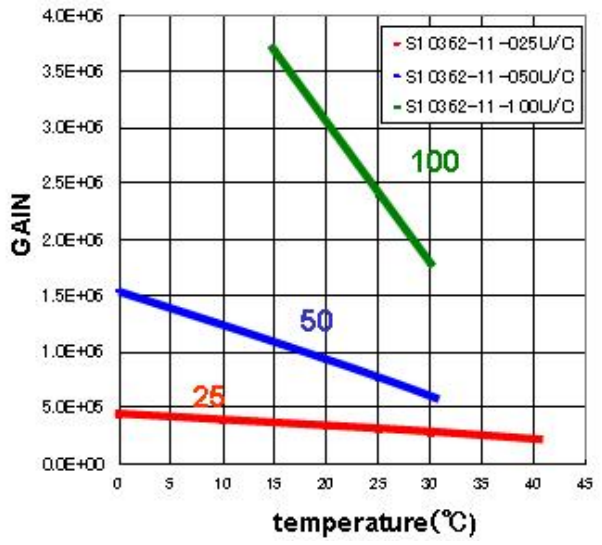

Fig.4 Gain vs. temperature



Fig.6 Timing resolution of $3 \mathrm{~mm} \square$-MPPC coupled with LYSO scintillator 


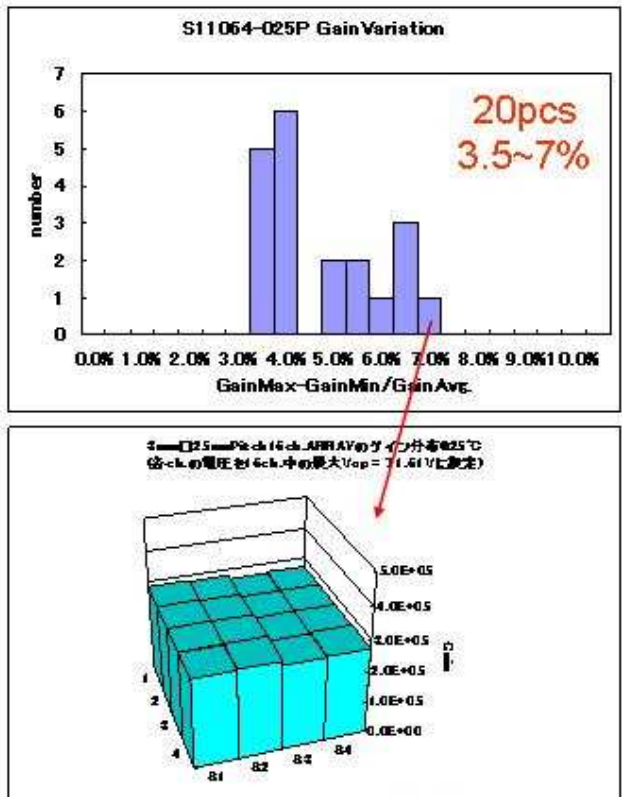

$\mathrm{S} 11064-025 \mathrm{P}(\mathrm{X} 1)$

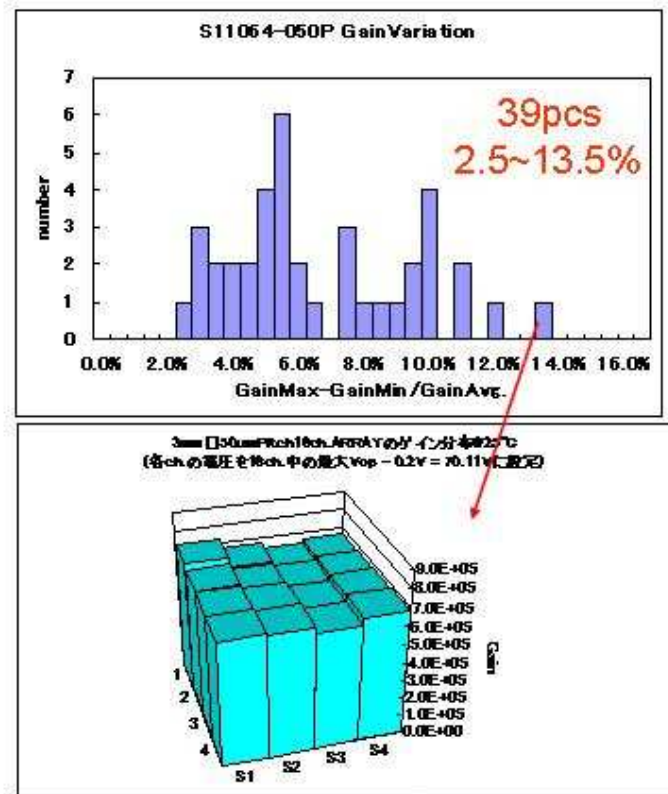

S11064-050P (X1)

Fig.7 Gain variation of 16ch-MPPC, 25um pixel type(left), 50um pixel type(right)

\section{Mass production of MPPC for T2K experiment}

S10362-13-050C (1.3mm $\square-50$ um pixel pitch) was adopted as photon sensor for $\mathrm{T} 2 \mathrm{~K}$ experiment. Figure 8 shows the photo of outline and the photo of housing provided by Kyoto University. Figure 9 shows the delivery history. From February in 2008 to February in 2009 we have delivered 63,885 pieces of MPPC smoothly. Figure 10 and 11 shows variation of Vop (voltage at gain $=7.5 \times 10^{5}$ ) and dark counts for all MPPC delivered. Vop was $70 \pm 2 \mathrm{~V}$ and variations are small. Dark count $1.3 \mathrm{Mcps}$ specifications are fully satisfied with good distribution.

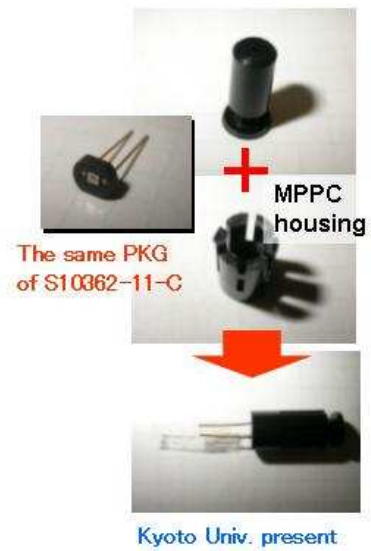

Fig.8 MPPC for T2K

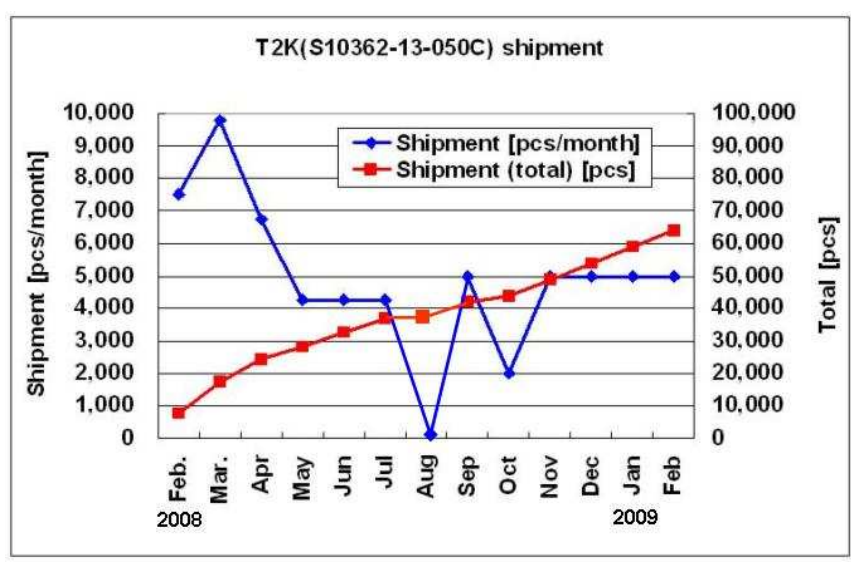

Fig.9 Shipment history of MPPCs for T2K 


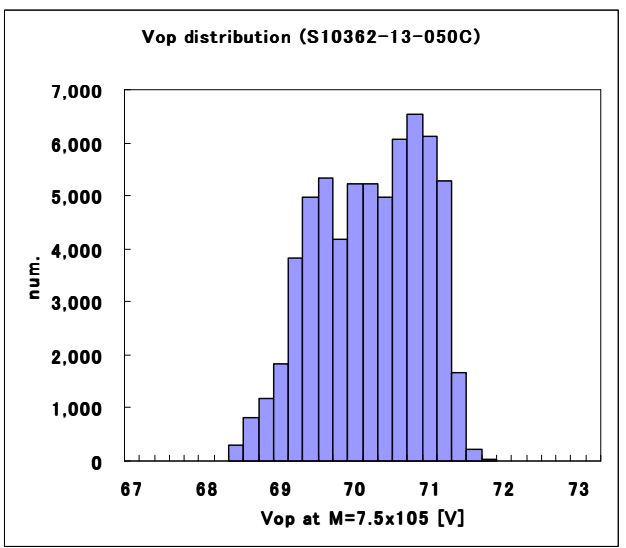

Fig.10 Vop distribution of MPPCs for $\mathrm{T} 2 \mathrm{~K}$

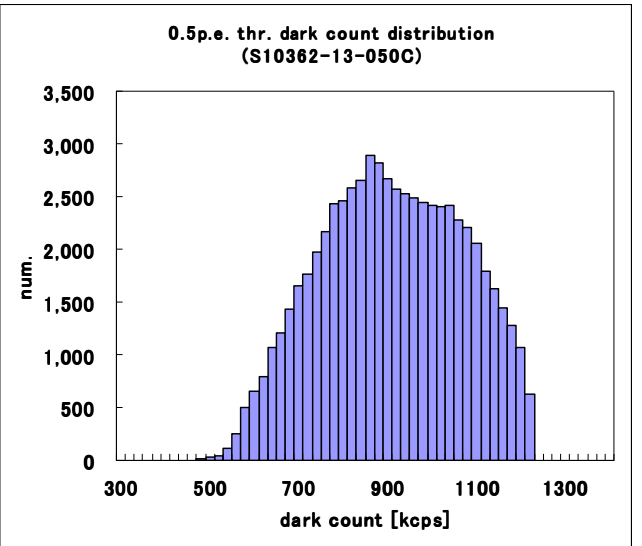

Fig.11 Dark count distribution of MPPCs for $\mathrm{T} 2 \mathrm{~K}$

\section{Timing resolution of MPPC}

Figure 12 shows the equivalent circuit and output pulse shape(red) derived from the equivalent circuit.[7] The Cq (parallel capacitance of quenching resistor) effect of spike-like first pulse (blue) has been introduced.[8 ] [9]

For improved timing resolution, we made samples and compared by focusing on the following points:

1. To rise faster. $\Rightarrow \mathrm{Cd}$ to reduce

2. To increase $\mathrm{Cq}$ effect for spike shape. $\Rightarrow$ Capacitance between pixel and readout tarce to increase

3. To reduce the variation of waveform between pixels $\Rightarrow$ to reduce the trace resistance Figure 13 shows the measurement setup of timing resolution. Single photon level is incident on MPPC and measure the time until the setting threshold. Measure 10K times and obtain the timing resolution by Gaussian fit of the histogram.
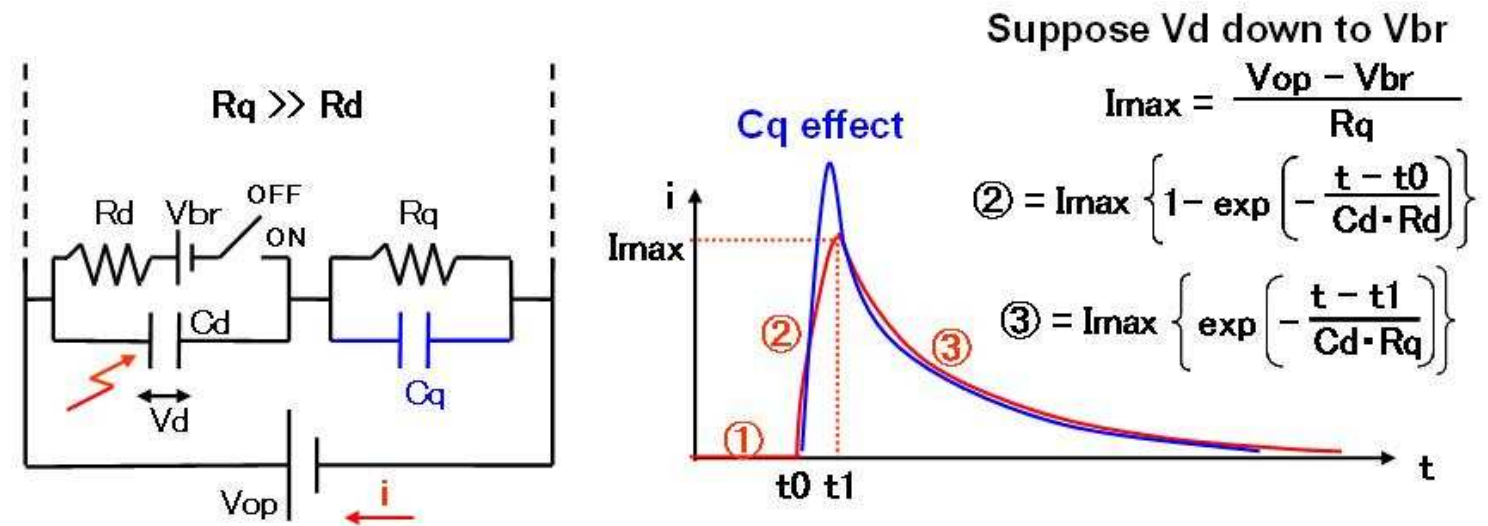

Fig.12 Equivalent circuit of MPPC (left) and pulse shape explanation of MPPC (right) 


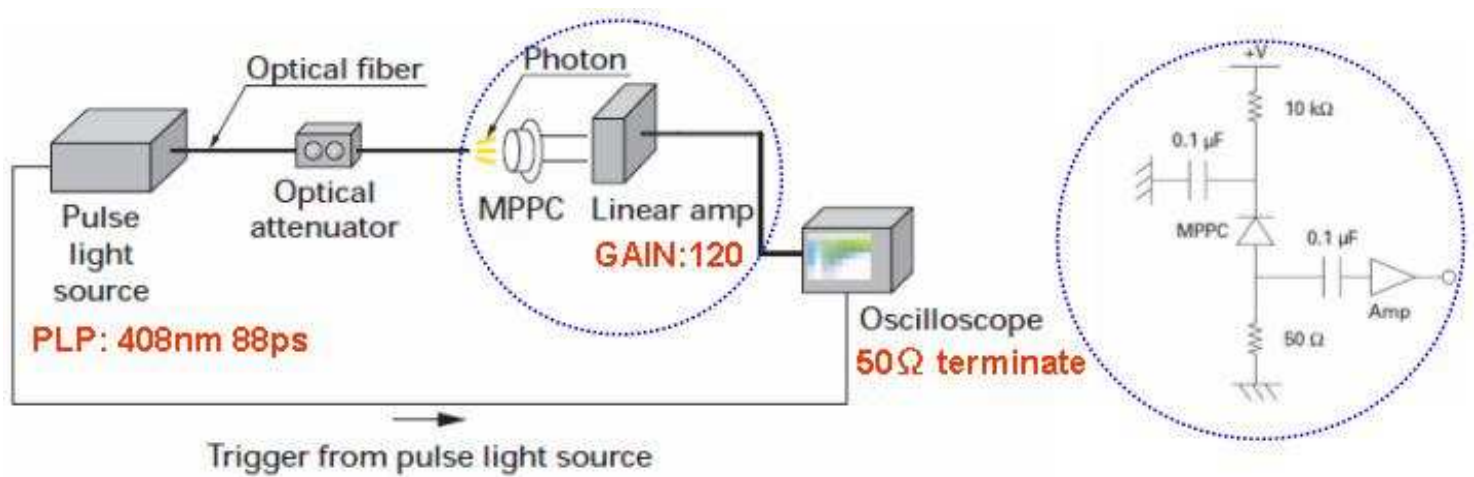

Fig.13 Measurement setup for timing resolution

Figure 14 shows picture of chip and experimental parameters of $1 \mathrm{~mm} \square-100$ um samples. STD is a traditional device. "Small pixel" is the smaller pixel size than that of "STD". "Wide trace" is the same pixel size of "Small pixel" but has wide trace electrode. Quenching resistance is obtained from the forward IV characteristics. Cd as the capacitance to affect the gain obtained from the linear bias dependence of gain. Ctotal is measured directly between the terminals. Cstray as the capacitance not to affect the gain obtained that Ctotal divided by the number of pixels minus the amount of $\mathrm{Cd}$. The gain of "Small pixel" and "Wide trace" are the same because $\mathrm{Cd}$ and $\Delta \mathrm{V}$ are the same, but the Cstray of "Wide trace" is bigger than that "Small pixel", so it can be said that Cstray by expanding the trace electrode dose not affect the gain.

Figure 15 shows the pulse shape and the threshold dependent of timing resolution of $1 \mathrm{~mm}$ $\square$-100um samples. "Wide trace" has spike shape and best timing resolution of around $315 \mathrm{pF}$ (FWHM) at $28.9 \mathrm{mV}$ threshold and believed to $\mathrm{Cq}$ effect. In this case, $\mathrm{Cq}$ is not the capacitance of quenching resistor but the capacitance between the pixel electrode and trace electrode. "Small pixel" has better timing resolution than "STD" due to smaller Cd. Figure 16 and 17 shows these of $1 \mathrm{~mm} \square$-50um samples, The trends are the same as those of $100 \mathrm{um}$ samples, and about 285ps (FWHM) was obtained at $16.5 \mathrm{mV}$ threshold for "Wide trace".

Figure 18 shows the result of pulse shape variation between pixels of $1 \mathrm{~mm} \square-50 \mathrm{um}$ samples. Laser light is incident on the center of the 21 pixels. Peak height and height ratio of $12 \mathrm{~ns}$ are obtained. Peak height of "Small pixel" is larger than "STD" and smaller than "Wide trace". Peak heights of "Wide trace" and "Small pixel" are smaller variation than "STD"(Left graph). Height ratio of "Wide trace" and "Small pixel" are smaller variation than "STD"(right graph). 


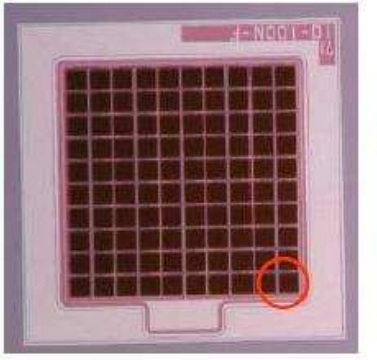

\begin{tabular}{|l|c|c|c|}
\hline Sample name & STD & Small pixel & Wide trace \\
\hline Fill factor & $78 \%$ & $72 \%$ & $72 \%$ \\
\hline$\Delta \mathrm{V}$ (Vop-Vbr) \#1 & $1.02 \mathrm{~V}$ & $1.18 \mathrm{~V}$ & $1.18 \mathrm{~V}$ \\
\hline Dark count at Vop & $1075 \mathrm{Kcps}$ & $1089 \mathrm{Kcps}$ & $1243 \mathrm{Kcps}$ \\
\hline Pixel capacitance (Cd) \#2 & $373 \mathrm{fF}$ & $323 \mathrm{fF}$ & $325 \mathrm{fF}$ \\
\hline Stray capacitance / pixel \#3 & $17 \mathrm{fF}$ & $37 \mathrm{fF}$ & $61 \mathrm{fF}$ \\
\hline PDE at Vop, 440nm & $79.7 \%$ & $76.2 \%$ & $77.6 \%$ \\
\hline
\end{tabular}

\#1: Vop is at $2.4 \mathrm{E} 06 \quad \# 2:$ by GAIN vs VR curve $\# 3:$ Ctotal $/ 100-\mathrm{Cd}$ at $25^{\circ} \mathrm{C}$

Fig.14 100um pitch samples pictures of pixel(upper), parameters(lower)

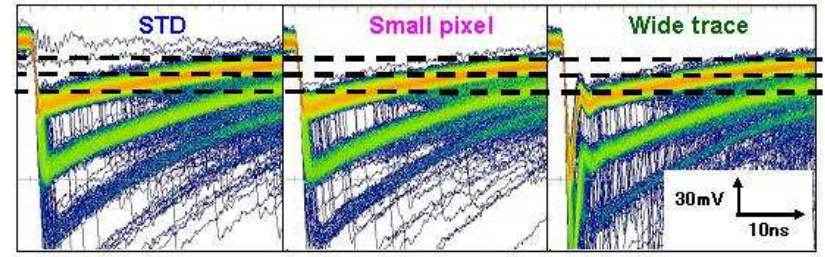

Fig.15 Pulse shape of 100um pitch each samples(upper) and measurement data of timing resolution of $1 \mathrm{p} . \mathrm{e}$. vs threshould(right)

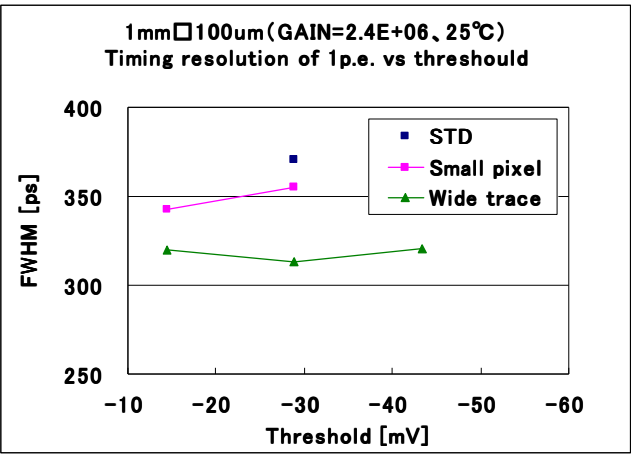

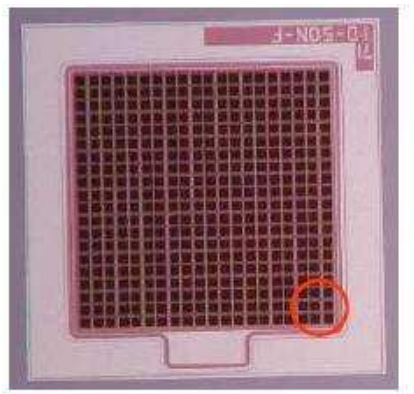

Quenching resistance $=130 \mathrm{~K} \Omega$ by forward IV curve
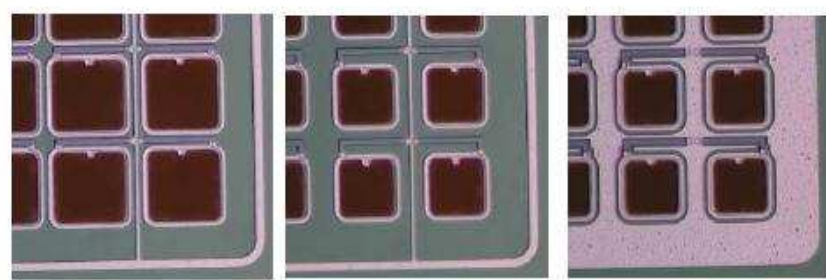

\begin{tabular}{|l|c|c|c|}
\hline Sample name & STD & Small pixel & Wide trace \\
\hline Fill factor & $62 \%$ & $38 \%$ & $38 \%$ \\
\hline$\Delta \mathrm{V}(\mathrm{Vop}-\mathrm{Vbr}) \quad \# 1$ & $1.31 \mathrm{~V}$ & $2.02 \mathrm{~V}$ & $2.01 \mathrm{~V}$ \\
\hline Dark count at Vop & $535 \mathrm{Kcps}$ & $484 \mathrm{Kcps}$ & $502 \mathrm{Kcps}$ \\
\hline Pixel capacitance (Cd) \#2 & $90 \mathrm{fF}$ & $59 \mathrm{fF}$ & $60 \mathrm{fF}$ \\
\hline Stray capacitance / pixel \#3 & $2.5 \mathrm{fF}$ & $11 \mathrm{fF}$ & $23 \mathrm{fF}$ \\
\hline PDE at Vop, 440nm & Not measure & Not measure & Not measure \\
\hline
\end{tabular}

\#1: Vop is at 7.5E05 \#2: by GAIN vs VR curve \#3: Ctotal $/ 400-\mathrm{Cd}$ at $25^{\circ} \mathrm{C}$

Fig.16 50um pitch samples pictures of pixel(upper), parameters(lower) 

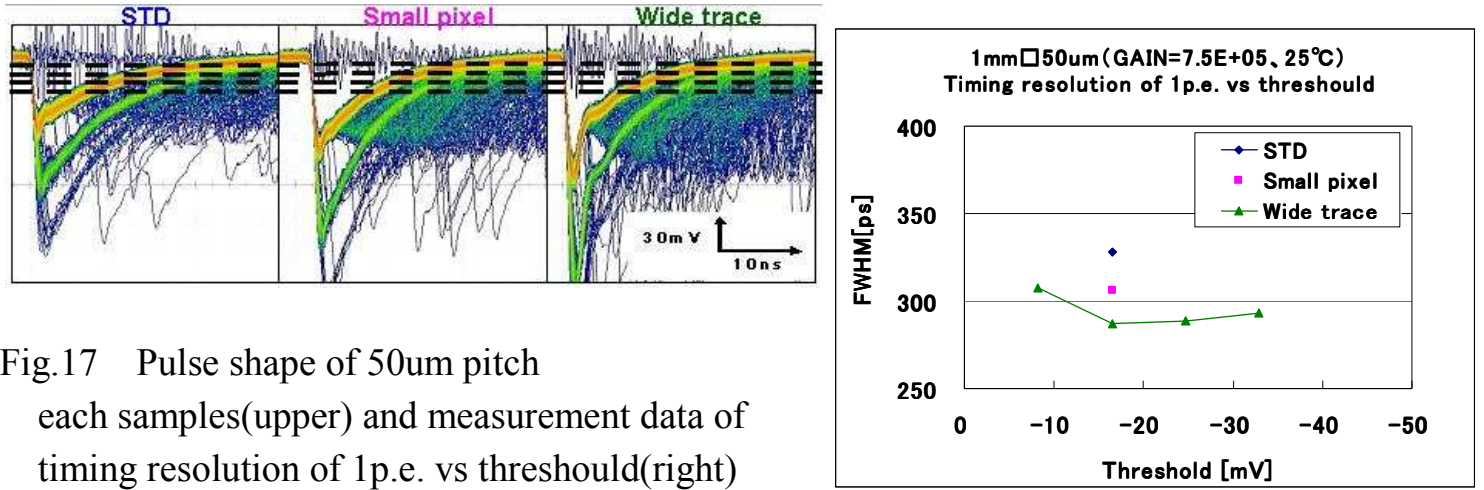

Fig.17 Pulse shape of 50um pitch each samples(upper) and measurement data of timing resolution of 1 p.e. vs threshould(right)
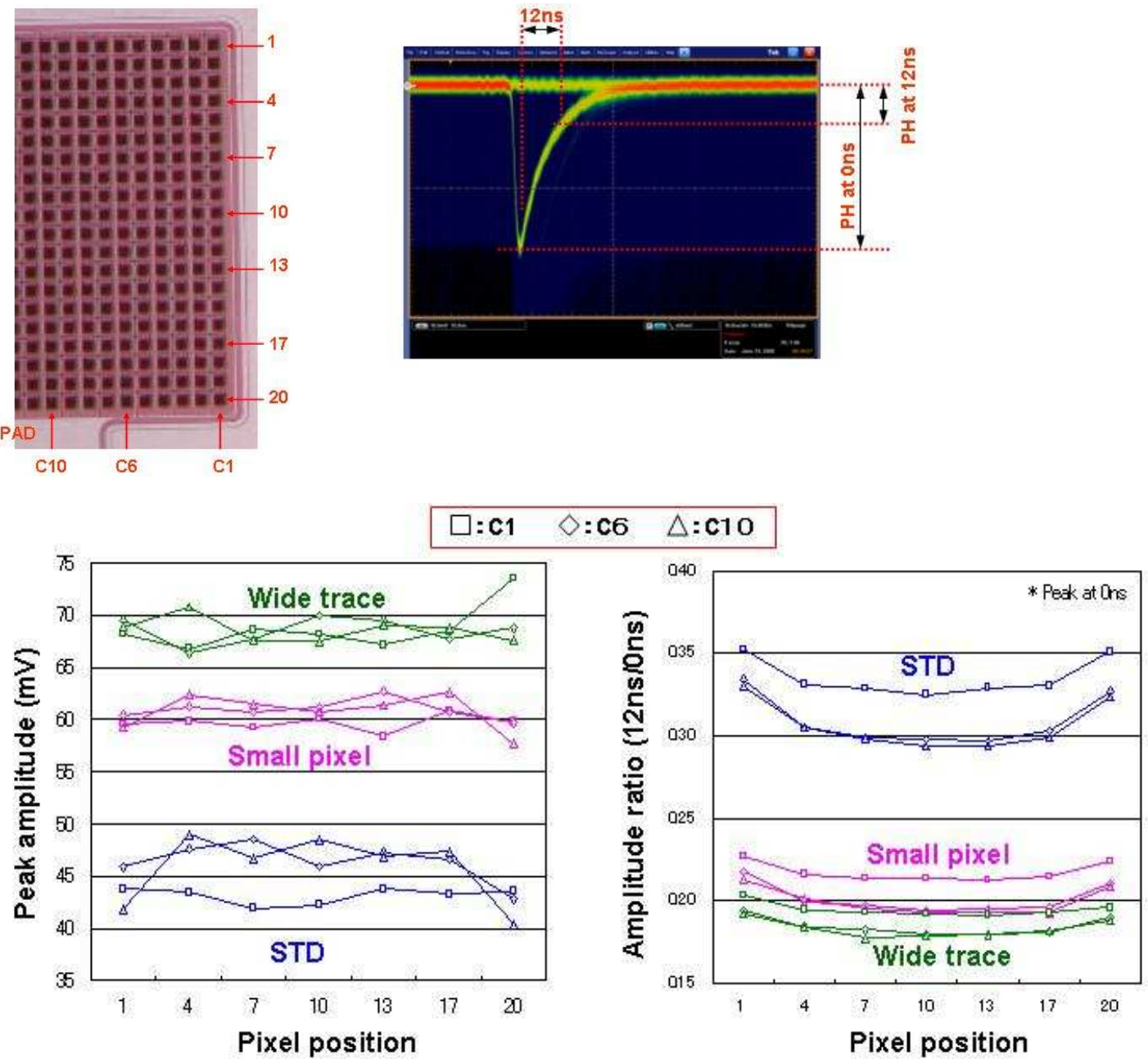

Fig18. Pulse shape uniformity of 50um pitch samples position number of pixels(upper left), Description of pulse shape(Upper-right) pulse height vs. pixel position(lower left), pulse decay vs. pixel position(lower right) 


\section{Conclusion}

We introduced line up of MPPCs, single channel type, array type, with a thermoelectrically cooler type. We explained the delivery history and variation of characteristics of $60 \mathrm{~K}$ pieces MPPCs adapted for T2K. We confirmed that "Wide trace" device is effective for improving the timing resolution.

\section{References}

[1] V. Golovin, V. Saveliev, "Novel type of avalanche photodetector with Geiger mode opration", NIM A $518(2004) 560$

[2] V. Saveliev, "The recent development and study of silicon photomultiplier", NIM A 535 (2004) 528

[3] A. N. Otte et al., "Prospects of Using Silicon Photomultipliers for the Astroparticle Physics Experiments EUSO and MAGIC“،, IEEE Trans. Nucl. Sci. 53 (2006) 636

[4] I. Britvitch et al., "Avalanche photodiodes now and possible developments", NIM A 535 (2004) 523

[5] K.Yamamoto et al., "Newly developed semiconductor detector by Hamamatsu",PD07 proceedings

[6] Hamamatsu Photonics, MPPC data sheet

[7] C. Piomonte et al., "A new Silicon Photomultiplier structure for blue light detection”, NIM A 568 (2006) 224-232

[8] F. Corsi et al.,."Modelling a silicon photomultiplier (SiPM) as a signal source for optimum frontend design”, NIM A 572 (2007) 416-418

[9] H.Otono et al., "On the basic mechanism of Pixelaized Photon Detectors", NIM A 50157 